\title{
Septic B-Spline Collocation Method for the Numerical Solution of the Modified Equal Width Wave Equation
}

\author{
Turabi Geyikli, Seydi Battal Gazi Karakoc \\ Department of Mathematics, Faculty of Education, Inonu University, Malatya, Turkey \\ E-mail:tgeyikli@inonu.edu.tr,sbgk44@mynet.com \\ Received March 25, 2011; revised April 20, 2011; accepted April 23, 2011
}

\begin{abstract}
Numerical solutions of the modified equal width wave equation are obtained by using collocation method with septic B-spline finite elements with three different linearization techniques. The motion of a single solitary wave, interaction of two solitary waves and birth of solitons are studied using the proposed method. Accuracy of the method is discussed by computing the numerical conserved laws error norms $L_{2}$ and $L_{\infty}$. The numerical results show that the present method is a remarkably successful numerical technique for solving the MEW equation. A linear stability analysis shows that this numerical scheme, based on a Crank Nicolson approximation in time, is unconditionally stable.
\end{abstract}

Keywords: Finite Element, Septic B-Spline, Collocation, Soliton, Solitary Waves

\section{Introduction}

This study is concerned with the numerical solution, using septic B-spline functions in collocation method, of the modified equal width wave (MEW) equation, which was introduced by Morrison et al. [1] as a model equation to describe the nonlinear dispersive waves. Few analytical solutions of the MEW equation are known. Thus numerical solutions of the MEW equation can be important and comparison between analytic solution can be made. Many methods have been proposed to solve the EW and MEW equation. Gardner and Gardner solved the EW equation with the Galerkin's method using cubic $\mathrm{B}$-splines as a trial and test function [2,3] and a Petrov-Galerkin method using quadratic B-spline element [4]. Zaki considered the solitary wave interactions for the MEW equation by Petrov-Galerkin method using quintic B-spline finite elements [5] and obtained the numerical solution of the EW equation by using least-squares method [6]. Wazwaz investigated the MEW equation and two of its variants by the tanh and the sine-cosine methods [7]. Esen applied a lumped Galerkin method based on quadratic B-spline finite element has been used for solving the EW and MEW equation [8,9]. Saka proposed algorithms for the numerical solution of the MEW equation using quintic B-spline collocation method [10]. A solution based on a collocation method incorporated cubic B-splines is investigated by Dağ and Saka [11].
Variational iteration method is introduced to solve the MEW equation by Junfeng Lu [12]. Hamdi et al. [13] derived exact solitary wave solutions of the generalized EW equation using Maple software. D. J. Evans and K. R. Raslan [14] studied the generalized EW equation by using collocation method based on quadratic B-splines to obtain the numerical solutions of a single solitary waves, and the birth of solitons.

The modified equal width wave equation which is as a model for non-linear dispersive waves, considered here has the normalized form [1]

$$
U_{t}+3 U^{2} U_{x}-\mu U_{x x t}=0
$$

with the physical boundary conditions $U \rightarrow 0$ as $x \rightarrow \pm \infty$, where $t$ is time and $x$ is the space coordinate, $\mu$ is a positive parameter. For this study boundary conditions are chosen

$$
\begin{aligned}
& U(a, t)=0, U(b, t)=0, \\
& U_{x}(a, t)=0, U_{x}(b, t)=0, \\
& U_{x x x}(a, t)=0, U_{x x x}(b, t)=0,
\end{aligned}
$$

and the initial condition as

$$
U(x, 0)=f(x), a \leq x \leq b
$$

where $f(x)$ is a localized disturbance inside the considered interval. 


\section{Septic B-Spline Collocation Method}

The interval $[a, b]$ is partitioned into uniformly sized finite elements by the knots $x_{i}$ such that $a=x_{0}<x_{1} \cdots<x_{N}=b$ and $h=\left(x_{i+1}-x_{i}\right)$. The septic B-splines $\phi_{i}(x),(i=-3(1) N+3)$, at the knots $x_{i}$ are defined over the interval $[a, b]$ as $[15]$,

$$
\begin{aligned}
& x \in\left[x_{i-4}, x_{i-3}\right], \\
& x \in\left[x_{i-3}, x_{i-2}\right], \\
& x \in\left[x_{i-2}, x_{i-1}\right], \\
& x \in\left[x_{i-1}, x_{i}\right], \\
& x \in\left[x_{i}, x_{i+1}\right], \\
& x \in\left[x_{i+1}, x_{i+2}\right], \\
& x \in\left[x_{i+2}, x_{i+3}\right], \\
& x \in\left[x_{i+3}, x_{i+4}\right], \\
& \text { otherwise. }
\end{aligned}
$$

The set of splines, $\left\{\phi_{-3}(x), \phi_{-2}(x), \cdots, \phi_{N+3}(x)\right\}$ forms a basis for functions defined over $[a, b]$. The numerical solution $U_{N}(x, t)$ to $U(x, t)$ takes the form

$$
U_{N}(x, t)=\sum_{i=-3}^{N+3} \delta_{i}(t) \phi_{i}(x)
$$

where $\delta_{i}$ are unknown, time dependent quantities to be determined from the boundary and collocation conditions and $\phi_{i}(x)$ are septic B-spline. Each septic B-spline covers 8 elements thus each element $\left[x_{i}, x_{i+1}\right]$ is covered by 8 splines. A typical finite interval $\left[x_{i}, x_{i+1}\right]$ is mapped to the interval $[0,1]$ by a local coordinate transformation defined by $h \xi=x-x_{i}, 0 \leq \xi \leq 1$. Therefore septic B-splines (3) in terms of $\xi$ over $[0,1]$ can be given as

$$
\begin{aligned}
& \phi_{i-3}=1-7 \xi+21 \xi^{2}-35 \xi^{3}+35 \xi^{4}-21 \xi^{5}+7 \xi^{6}-\xi^{7} \\
& \phi_{i-2}=120-392 \xi+504 \xi^{2}-280 \xi^{3}+84 \xi^{5}-42 \xi^{6}+7 \xi^{7} \\
& \phi_{i-1}=1191-1715 \xi+315 \xi^{2}+665 \xi^{3}-315 \xi^{4}-105 \xi^{5}+105 \xi^{6}-21 \xi^{7} \\
& \phi_{i}=2416-1680 \xi+560 \xi^{4}-140 \xi^{6}+35 \xi^{7} \\
& \phi_{i+1}=1191+1715 \xi+315 \xi^{2}-665 \xi^{3}-315 \xi^{4}+105 \xi^{5}+105 \xi^{6}-35 \xi^{7} \\
& \phi_{i+2}=120+392 \xi+504 \xi^{2}+280 \xi^{3}-84 \xi^{5}-42 \xi^{6}+21 \xi^{7} \\
& \phi_{i+3}=1+7 \xi+21 \xi^{2}+35 \xi^{3}+35 \xi^{4}+21 \xi^{5}+7 \xi^{6}-7 \xi^{7} \\
& \phi_{i+4}=\xi^{7} .
\end{aligned}
$$

Since all splines apart from $\phi_{i-3}(x), \phi_{i-2}(x), \cdots, \phi_{i+3}(x)$ are zero over the element $[0$, $1]$. For the problem the finite elements are identified with the interval $\left[x_{i}, x_{i+1}\right]$. Using the nodal values $U_{i}, U_{i}^{\prime}, U_{i}^{\prime \prime}$ and $U_{i}^{\prime \prime \prime}$ are given in terms of the parameter $\delta_{i}$ by:

$$
\begin{aligned}
& U_{i}=\delta_{i+3}+120 \delta_{i+2}+1191 \delta_{i+1}+2416 \delta_{i}+1191 \delta_{i-1}+120 \delta_{i-2}+\delta_{i-3}, \\
& h U_{i}^{\prime}=7\left(\delta_{i+3}+56 \delta_{i+2}+245 \delta_{i+1}-245 \delta_{i-1}-56 \delta_{i-2}-\delta_{i-3}\right), \\
& h^{2} U_{i}^{\prime \prime}=42\left(\delta_{i+3}+24 \delta_{i+2}+15 \delta_{i+1}-80 \delta_{i}+15 \delta_{i-1}+24 \delta_{i-2}+\delta_{i-3}\right), \\
& h^{3} U_{i}^{\prime \prime \prime}=210\left(\delta_{i+3}+8 \delta_{i+2}-19 \delta_{i+1}+19 \delta_{i-1}-8 \delta_{i-2}-\delta_{i-3}\right),
\end{aligned}
$$

and the variation of $U$ over the element $\left[x_{i}, x_{i+1}\right]$ is given by

$$
U=\sum_{i=-3}^{N+3} \delta_{i} \phi_{i}
$$

We now identify the collocation points with the knots and use (6) to evaluate $U_{i}$ and its space derivatives in (1). This leads to a set of ordinary differential equations of the form 


$$
\begin{aligned}
& \dot{\delta}_{i+3}+120 \dot{\delta}_{i+2}+1191 \dot{\delta}_{i+1}+2416 \dot{\delta}_{i}+1191 \dot{\delta}_{i-1}+120 \dot{\delta}_{i-2}+\dot{\delta}_{i-3}-21 \frac{1}{h} Z_{i}\left(\delta_{i+3}+56 \delta_{i+2}+245 \delta_{i+1}-245 \delta_{i-1}-56 \delta_{i-2}-\delta_{i-3}\right) \\
& -42 \frac{\mu}{h^{2}}\left(\delta_{i+3}+24 \delta_{i+2}+15 \delta_{i+1}-80 \delta_{i}+15 \delta_{i-1}+24 \delta_{i-2}+\delta_{i-3}\right)=0,
\end{aligned}
$$

where

$$
Z_{i}=\left(\delta_{i+3}+120 \delta_{i+2}+1191 \delta_{i+1}+2416 \delta_{i}+1191 \delta_{i-1}+120 \delta_{i-2}+\delta_{i-3}\right)^{2} .
$$

If time parameters $\delta_{i}$ and its time derivatives $\dot{\delta}_{i}$ in (7) are discretized by the Crank-Nicolson formula and usual finite difference approximation, respectively:

$$
\delta_{i}=\frac{1}{2}\left(\delta^{n}+\delta^{n+1}\right), \dot{\delta}_{i}=\frac{\delta^{n+1}-\delta^{n}}{\Delta t}
$$

We obtain a recurrence relationship between two time levels $n$ and $n+1$ relating two unknown parameters $\delta_{i}^{n+1}, \delta_{i}^{n}$

$$
\begin{aligned}
& \gamma_{i 1} \delta_{i+3}^{n+1}+\gamma_{i 2} \delta_{i+2}^{n+1}+\gamma_{i 3} \delta_{i+1}^{n+1}+\gamma_{i 4} \delta_{i}^{n+1}+\gamma_{i 5} \delta_{i-1}^{n+1}+\gamma_{i 6} \delta_{i-2}^{n+1}+\gamma_{i 7} \delta_{i-3}^{n+1} \\
& =\gamma_{i 7} \delta_{i+3}^{n}+\gamma_{i 6} \delta_{i+2}^{n}+\gamma_{i 5} \delta_{i+1}^{n}+\gamma_{i 4} \delta_{i}^{n}+\gamma_{i 3} \delta_{i-1}^{n}+\gamma_{i 2} \delta_{i-2}^{n}+\gamma_{i 1} \delta_{i-3}^{n}
\end{aligned}
$$

where

$$
\begin{aligned}
& \gamma_{i 1}=\left(1+E Z_{i}-M\right), \gamma_{i 2}=\left(120+56 E Z_{i}-24 M\right), \gamma_{i 3}=\left(1191+245 E Z_{i}-15 M\right), \\
& \gamma_{i 4}=(2416+80 M), \gamma_{i 5}=\left(1191-245 E Z_{i}-15 M\right), \gamma_{i 6}=\left(120-56 E Z_{i}-24 M\right), \\
& \gamma_{i 7}=\left(1-E Z_{i}-M\right) \quad i=0,1, \cdots, N, \\
& E=\frac{21}{2 h} \Delta t, \quad M=\frac{42}{h^{2}} \mu .
\end{aligned}
$$

For the first linearization, we suppose that the quantity $U$ in the non-linear term $U^{2} U_{x}$ to be locally constant. This is equivalent to assuming that in (7) all $Z_{i}$ are equal to a local constant. Furthermore, we can write the nonlinear term

$$
U^{2} U_{x}=U U U_{x}
$$

and apply the Rubin and Graves [16] linearization technique

$$
\left(U U_{x}\right)^{n+1}=U^{n+1} U_{x}+U^{n} U_{x}^{n+1}-U^{n} U_{x}^{n}
$$

to the $U U_{x}$ term in (8) and we can also apply the Caldwell and Smith [17] linearization technique

$$
\left(U U_{x}\right)^{n+1}=\frac{1}{2}\left(U^{n+1} U_{x}+U^{n} U_{x}^{n+1}\right)
$$

to the $U U_{x}$ term in (8). The system (8) consists of $N+1$ linear equation in $N+7$ unknowns $\left(\delta_{-3}, \delta_{-2}, \cdots, \delta_{N+2}, \delta_{N+3}\right)^{\mathrm{T}}$. To obtain a unique solution to this system we need 6 additional constraints. These are obtained from the boundary conditions and can be used to eliminate $\delta_{-3}, \delta_{-2}, \delta_{-1}$ and $\delta_{N+1}, \delta_{N+2}, \delta_{N+3}$ from the set (2) which then becomes a matrix equation for the $N+1$ unknowns $\boldsymbol{\delta}=\left(\delta_{0}, \delta_{1}, \cdots, \delta_{N}\right)^{\mathrm{T}}$ of the form $A \delta^{n+1}=B \delta^{n}$. The matrices $A$ and $B$ are septa-diagonal $(N+1) \times(N+1)$ matrices and so are easily solved by septa-diagonal algorithm.

\section{Initial State}

To start evolution of the vector of parameters $\delta^{n}, \delta^{0}$ can be determined from the boundary conditions and the initial condition $U(x, 0)$. So we can rewrite approximation (4) for the initial condition

$$
U_{N}(x, 0)=\sum_{i=-3}^{N+3} \delta_{i}(0) \phi_{i}(x)
$$

where parameters $\delta_{i}^{0}$ will be determined. To determine the parameters $\delta^{0}=\left(\delta_{-3}^{0}, \delta_{-2}^{0}, \cdots, \delta_{N+2}^{0}, \delta_{N+3}^{0}\right)$, we require the initial numerical approximation $U_{N}(x, 0)$ to satisfy the following conditions:

1) it must agree with the exact initial condition $U(x, 0)$ at the knots $x_{i}$.

2) the first, second and third derivatives of the approximate initial condition agree with those of the exact initial conditions at both ends of the range. These two conditions can be expressed as:

$$
\begin{aligned}
& U_{N}(x, 0)=U_{N}\left(x_{i}, 0\right), 0 \leq i \leq N, \\
& U_{N x}(a, 0)=U_{N x}(b, 0)=0, \\
& U_{N x x}(a, 0)=U_{N x x}(b, 0)=0, \\
& U_{N x x x}(a, 0)=U_{N x x x}(b, 0)=0 .
\end{aligned}
$$

The above conditions lead to $K \delta^{0}=b$ matrix equation, which is solved by using a variant of Thomas algorithm. 


$$
\boldsymbol{K}=\left[\begin{array}{ccccccc}
1536 & 2712 & 768 & 24 & & \\
\frac{82731}{81} & \frac{210568.5}{81} & \frac{104796}{81} & \frac{10063.5}{81} & 1 & \\
\frac{9600}{81} & \frac{96597}{81} & \frac{195768}{81} & \frac{96474}{81} & 120 & 1 \\
1 & 120 & 1191 & 2416 & 1191 & 120 \\
& & & & &
\end{array}\right.
$$$$
\boldsymbol{\delta}^{0}=\left(\delta_{0}, \delta_{1}, \delta_{2}, \cdots, \delta_{N-2}, \delta_{N-1}, \delta_{N}\right)^{\mathrm{T}}
$$

and

$$
b=\left(U\left(x_{0}, 0\right), U\left(x_{1}, 0\right), \cdots, U\left(x_{N-1}, 0\right), U\left(x_{N}, 0\right)\right)^{\mathrm{T}}
$$

\section{Stability Analysis}

The stability analysis will be based on the von Neumann theory in which the growth factor of a typical Fourier mode

$$
\delta_{j}^{n}=\hat{\delta}^{n} \mathrm{e}^{i j k h},
$$

where $k$ is the mode number and $h$ the element size, is determined for a linearisation of the numerical scheme. Substituting the Fourier mode (13) into the linearised recurrence relationship (8) shows that the growth factor for $\bmod k$ is

$$
g=\frac{a-i b}{a+i b}
$$

where

$$
\begin{aligned}
a= & 1208+40 M-3(-397+5 M) \cos [h k] \\
& -24(-5+M) \cos [2 h k]+(1-M) \cos [3 h k] \\
b= & 245 E Z_{i} \sin [h k]+56 E Z_{i} \sin [2 h k]+E Z_{i} \sin [3 h k] .
\end{aligned}
$$

The modulus of $|g|$ is 1 therefore the linearised scheme is unconditionally stable.

\section{Numerical Examples and Results}

All computations were executed on a pentium $4 \mathrm{PC}$ in the Fortran code using double precision arithmetic. The conservation properties of (1) will be examined by calculating the lowest three invariants given as

$$
\begin{aligned}
& C_{1}=\int_{a}^{b} U \mathrm{~d} x \cong h \sum_{J=1}^{N} U_{j}^{n}, \\
& C_{2}=\int_{a}^{b}\left[U^{2}+\mu\left(U_{x}\right)^{2}\right] \mathrm{d} x=h \sum_{J=1}^{N}\left(U_{j}^{n}\right)^{2}+\mu\left(U_{x}\right)_{j}^{n}, \\
& C_{3}=\int_{a}^{b} U^{4} \mathrm{~d} x=h \sum_{J=1}^{N}\left(U_{j}^{n}\right)^{4},
\end{aligned}
$$

$$
\left.\begin{array}{cccccc}
1 & 120 & \frac{96474}{81} & \frac{195768}{81} & \frac{96597}{81} & \frac{9600}{81} \\
1 & \frac{10063.5}{81} & \frac{104796}{81} & \frac{210568.5}{81} & \frac{82731}{81} \\
& & 24 & 768 & 2712 & 1536
\end{array}\right]
$$

which correspond to mass, momentum and energy respectively [5]. The accuracy of the method is measured by both the error norm

$$
L_{2}=\left\|U^{\text {exact }}-U_{N}\right\|_{2}=\sqrt{h \sum_{J=0}^{N}\left|U_{j}^{\text {exact }}-\left(U_{N}\right)_{j}\right|^{2}},
$$

and the error norm

$$
L_{\infty}=\left\|U^{\text {exact }}-U_{N}\right\|_{\infty}=\max _{j}\left|U_{j}^{\text {exact }}-\left(U_{N}\right)_{j}\right| .
$$

To implement the method, three test problems: motion of a single solitary wave, interaction of two solitary waves and the maxwellian initial condition will be considered.

\section{Motion of Single Solitary Wave}

For this problem, we consider Equation (1) with the boundary conditions $U \rightarrow 0$ as $x \rightarrow \pm \infty$ and the initial condition

$$
U(x, 0)=A \sec h\left(k\left[x-x_{0}\right]\right) .
$$

This problem has an exact solution of the form

$$
U(x, t)=A \operatorname{sech}\left(k\left[x-x_{0}-v t\right]\right)
$$

which represents the motion of a single solitary wave with amplitude $A$, here the wave velocity $v=A^{2} / 2$ and $k=\sqrt{1 / \mu}$. For this problem the analytical values of the invariants are [5]

$$
C_{1}=\frac{A \pi}{k}, C_{2}=\frac{2 A^{2}}{k}+\frac{2 \mu k A^{2}}{3}, C_{3}=\frac{4 A^{4}}{3 k} .
$$

The analytical values of invariants are obtained from (1) $C_{1}=0.7853982, C_{2}=0.1666667, C_{3}=0.0052083$. For the numerical simulation of the motion of a single solitary wave, we have used the parameters $h=0.1$, $\Delta t=0.05, \mu=1, x_{0}=30, A=0.25$ through the inter- 
val $0 \leq x \leq 80$. The computations are done until time $t=20$ and in this lenght we find error norms $L_{2}, L_{\infty}$ and numerical invariants $C_{1}, C_{2}, C_{3}$ at various times. In Table 1 we compare the values of the invariants and error norms obtained using the present method with the three different approximation and the results of $[5,14$, $18,19]$ at different times. We can easily see from the this table that the error norms $L_{2}$ and $L_{\infty}$ are obtained sufficiently small and the quantities in the variants remain almost constant during the computer run for the first and second linearization techniques but we can not say the same for the third linearization technique. For the first and second linearization, the numerical values of invariants are $C_{1}=0.7853966, C_{2}=0.1667641, C_{3}=0.0052083$ and for the third linearization numerical values of invariants are $C_{1}=0.7855405, C_{2}=0.1667641, C_{3}=0.0052144$ at the $t=20$. Figure 1 shows that the proposed method perform the motion of propagation of a solitary wave satisfactorily, which moved to the right at a constant speed and preserved its amplitude and shape with in- creasing time as expected. Amplitude is 0.249999 at $t=0$ which is located at $x=30$, while it is 0.249922 at $t=20$ which is located at $x=30.6$. The absolute difference in amplitudes at times $t=0$ and $t=20$ is $7.7 \times 10^{-5}$ so that there is a little change between amplitudes.

\section{Interaction of Two Solitary Waves}

For this problem, we consider (1) with boundary conditions $U \rightarrow 0$ as $x \rightarrow \pm \infty$, interaction of two positive solitary waves is studied by using the initial condition

$$
U(x, 0)=\sum_{j=1}^{2} A_{j} \operatorname{sech}\left(k\left[x-x_{j}\right]\right) .
$$

where $k=\sqrt{1 / \mu}$.

We first used the parameters $h=0.1, \Delta t=0.025$, $\mu=1, A_{1}=1, A_{2}=0.5, \quad x_{1}=15, x_{2}=30$ through the interval $0 \leq x \leq 80$ which is used by Zaki [5]. These parameters provide solitary waves of magnitudes 1 and 0.5

Table 1. Invariants and error norms for single solitary waves.

\begin{tabular}{|c|c|c|c|c|c|c|}
\hline$t$ & Lineerization & $C_{1}$ & $C_{2}$ & $C_{3}$ & $L_{2} \times 10^{3}$ & $L_{\infty} \times 10^{3}$ \\
\hline 0 & & 0.7853966 & 0.1666664 & 0.0052083 & 0.0000000 & 0.0000000 \\
\hline 5 & & 0.7853966 & 0.1666664 & 0.0052083 & 0.0000979 & 0.0000622 \\
\hline 10 & First & 0.7853966 & 0.1666664 & 0.0052083 & 0.0002113 & 0.0001368 \\
\hline 15 & & 0.7853966 & 0.1666664 & 0.0052083 & 0.0003432 & 0.0002251 \\
\hline 20 & & 0.7853966 & 0.1666664 & 0.0052083 & 0.0004969 & 0.0003309 \\
\hline 0 & & 0.7853966 & 0.1666664 & 0.0052083 & 0.0000000 & 0.0000000 \\
\hline 5 & & 0.7853966 & 0.1666664 & 0.0052083 & 0.0000972 & 0.0000627 \\
\hline 10 & Second & 0.7853966 & 0.1666664 & 0.0052083 & 0.0002102 & 0.00001378 \\
\hline 15 & & 0.7853966 & 0.1666664 & 0.0052083 & 0.0003419 & 0.0002272 \\
\hline 20 & & 0.7853966 & 0.1666664 & 0.0052083 & 0.0004957 & 0.0003331 \\
\hline 0 & & 0.7853966 & 0.1666664 & 0.0052083 & 0.0000000 & 0.0000000 \\
\hline 5 & & 0.7854325 & 0.1666908 & 0.0052098 & 0.0237333 & 0.0228190 \\
\hline 10 & Third & 0.7854685 & 0.1667152 & 0.0052114 & 0.0480311 & 0.0454089 \\
\hline 15 & & 0.7855045 & 0.1667397 & 0.0052129 & 0.0734307 & 0.0678603 \\
\hline 20 & & 0.7855405 & 0.1667641 & 0.0052144 & 0.1004249 & 0.0900579 \\
\hline $20[5]$ & & 0.785397 & 0.166667 & 0.005210 & 0.0034500 & 0.0020300 \\
\hline 20 [14] & & 0.7849545 & 0.1664765 & 0.0051995 & 0.2498925 & 0.2905166 \\
\hline 20 [18] & & 0.7853977 & 0.1664735 & 0.0052083 & 0.2692812 & 0.2569972 \\
\hline 20 [19] & & - & - & - & 0.1958878 & 0.1744330 \\
\hline
\end{tabular}



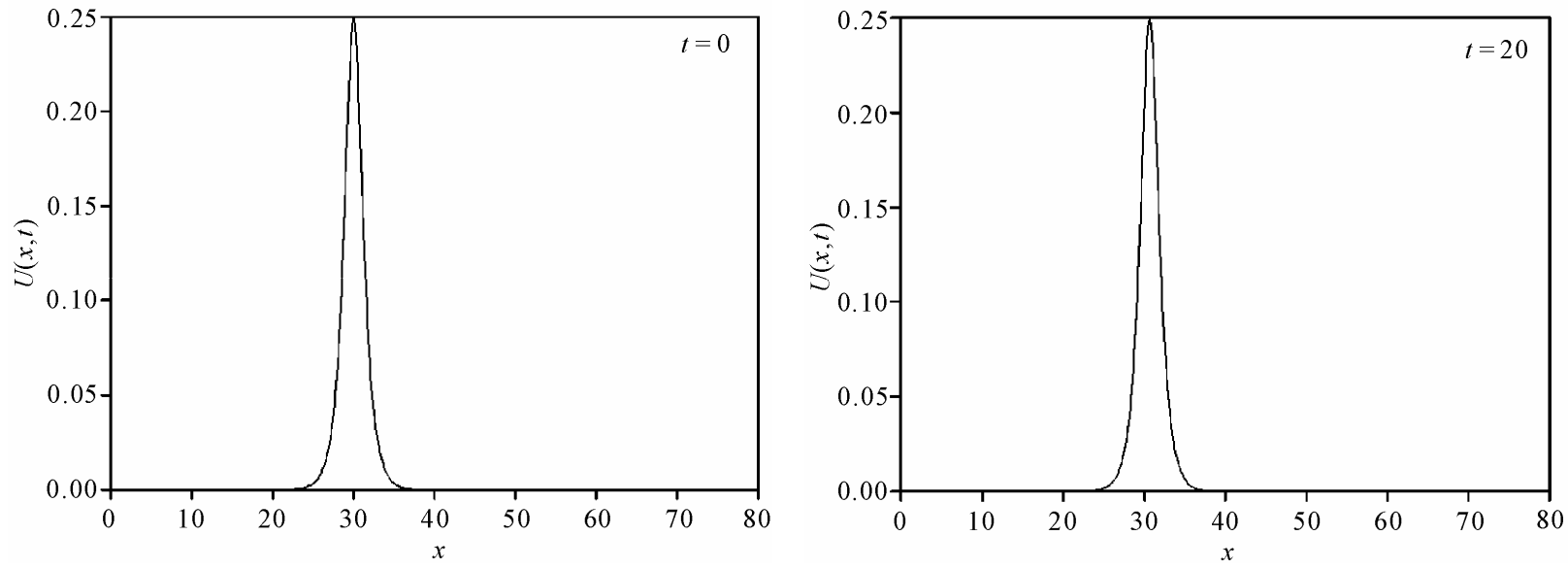

Figure 1. The motion of a single solitary wave.

and peak positions of them are located at $x=15$ and 30 . The analytical invariants are [14]

$$
\begin{aligned}
& C_{1}=\pi\left(A_{1}+A_{2}\right)=4.7123889, \\
& C_{2}=\frac{8}{3}\left(A_{1}^{2}+A_{2}^{2}\right)=3.3333333, \\
& C_{3}=\frac{4}{3}\left(A_{1}^{4}+A_{2}^{4}\right)=1.4166667 .
\end{aligned}
$$

Calculation is carried out with the time step

$\Delta t=0.025$ and space step $h=0.1$ over the region $0 \leq x \leq 80$. The experiment was run from $t=0$ to $t=55$ to allow the interaction to take place. Figure 2 shows the interaction of two positive solitary waves. It can be seen that at $t=5$ the wave with larger amplitude is on the left of the second wave with smaller amplitude. The larger wave catches up with the smaller one as time increases. Interaction started at about time $t=25$, overlapping processes occurred between times $t=25$ and 40 and waves started to resume their original shapes after time $t=40$. For the first and second linearization techniques at $t=55$, the amplitude of larger waves is 1.000149 at the point $x=44.4$ whereas the amplitude of the smaller one is 0.507317 at the point $x=34.6$. It is found that the absolute difference in amplitude is $7.3 \times 10^{-3}$ for the smaller wave and $0.149 \times 10^{-3}$ for the larger wave for this algorithm. For the third linearization technique at $t=55$, the amplitude of larger waves is 0.995933 at the point $x=44.7$ whereas the amplitude of the smaller one is 0.507477 at the point $x=34.6$. It is found that the absolute difference in amplitude is $7.4 \times 10^{-3}$ for the smaller wave and $4 \times 10^{-3}$ for the larger wave for this algorithm. In Table 2 we compares values invariants of the two solitary waves with results from first, second and third linearization. We see from the Table 2 that for the first and second linearization techniques, all 3 invariants are conserved by less than
$9.9 \times 10^{-5}$ during the experiment. Thus we have found

\begin{tabular}{|c|c|c|c|c|}
\hline \multicolumn{5}{|c|}{$A_{1}=1, A_{2}=0.5$} \\
\hline$t$ & Lineerization & $C_{1}$ & $C_{2}$ & $C_{3}$ \\
\hline 0 & & 4.7123733 & 3.3333294 & 1.4166643 \\
\hline 5 & & 4.7123660 & 3.3333183 & 1.4166532 \\
\hline 15 & & 4.7123494 & 3.3332959 & 1.4166308 \\
\hline 25 & First & 4.7123331 & 3.3332741 & 1.4166083 \\
\hline 35 & & 4.7123243 & 3.3335335 & 1.4165818 \\
\hline 45 & & 4.7123127 & 3.3332470 & 1.4165824 \\
\hline 55 & & 4.7122960 & 3.3332247 & 1.4165605 \\
\hline 0 & & 4.7123733 & 3.3333294 & 1.4166643 \\
\hline 5 & & 4.7123696 & 3.3333160 & 1.4166509 \\
\hline 15 & & 4.7123602 & 3.3332892 & 1.4166241 \\
\hline 25 & Second & 4.7123494 & 3.3332603 & 1.4165947 \\
\hline 35 & & 4.7123274 & 3.3332088 & 1.4165317 \\
\hline 45 & & 4.7123380 & 3.3332270 & 1.4165618 \\
\hline 55 & & 4.7123291 & 3.3332012 & 1.4165363 \\
\hline 0 & & 4.7123733 & 3.3333294 & 1.4166643 \\
\hline 5 & & 4.7318586 & 3.3857414 & 1.4689535 \\
\hline 15 & & 4.7738238 & 3.5004328 & 1.5869329 \\
\hline 25 & Third & 4.8195659 & 3.6284390 & 1.7245060 \\
\hline 35 & & 4.8528439 & 3.7197153 & 1.8236340 \\
\hline 45 & & 4.9055278 & 3.8734565 & 2.0013048 \\
\hline 55 & & 4.9694524 & 4.0643034 & 2.2347812 \\
\hline
\end{tabular}
that the conservation quantities are satisfactorily constant

Table 2. Invariants for interaction of two solitary wave. 

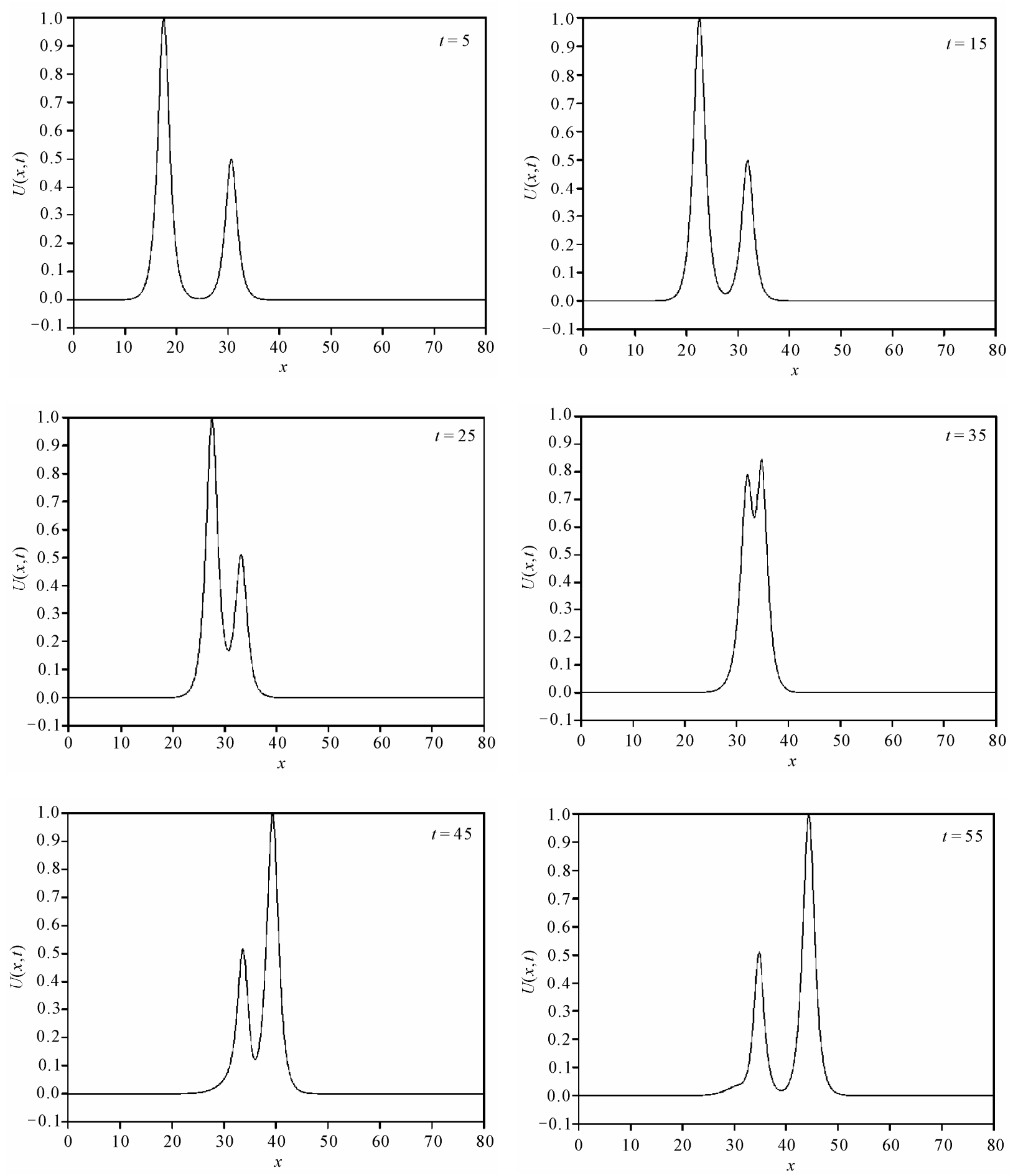

Figure 2. Interaction of two solitary waves at different times.

with the proposed algorithm.

We have also studied the interaction of two solitary waves with the following parameters: $\mu=1, x_{1}=15$, $x_{2}=30, A_{1}=-2, A_{2}=1$, together with time step $\Delta t=0.025$ and space step $h=0.1$ in the range $0 \leq x \leq$ 150. The experiment was run from $t=0$ to $t=55$ to allow the interaction to take place. Figure 3 shows the development of the solitary wave interaction.

As is seen from the Figure 3, at $t=0$ a wave with the negative amplitude is on the left of another wave with the positive amplitude. The larger wave with the negative amplitude catches up with the smaller one with 

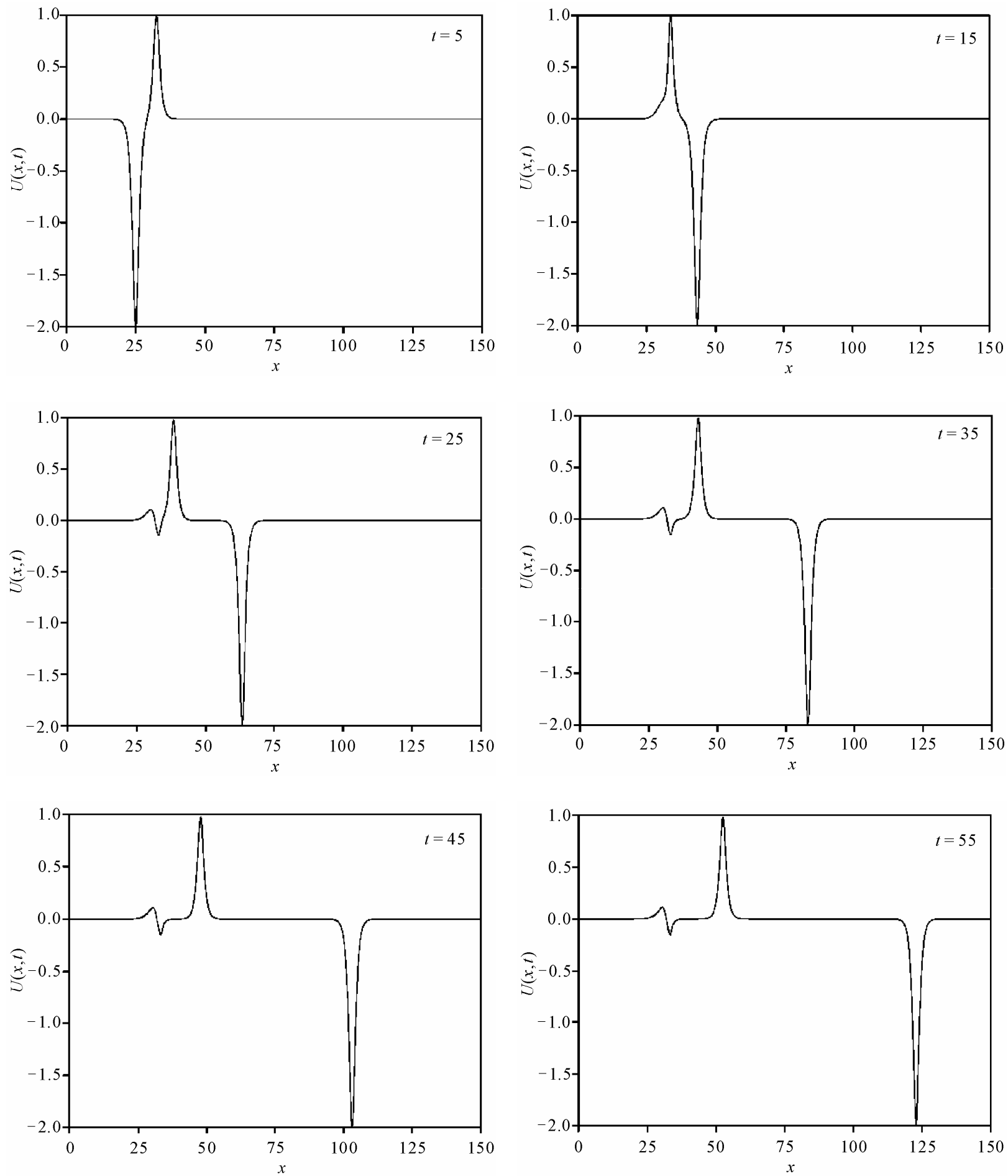

Figure 3. Interaction of two solitary waves at different times.

the positive amplitude as the time increases. For the first linearization technique at $t=55$, the amplitude of the smaller wave is 0.974695 at the point $x=52.5$, whereas the amplitude of the larger one is -1.989036 at the point $x=122.8$. For the second linearization technique at $t=55$, the amplitude of the smaller wave is 0.972778 at the point $x=52.5$, whereas the amplitude of the larger one is -1.986701 at the point $x=122.8$. It is found that the absolute difference in amplitudes is $0.253 \times 10^{-1}, 0.272 \times 10^{-1}$ for the smaller wave and $0.133 \times 10^{-1}, 0.109 \times 10^{-1}$ for the larger wave, respectively. 
The analytical invariants by using Equation (1) can be found as $C_{1}=-3.1415927, C_{2}=13.3333333$,

$C_{3}=22.6666667$. Table 3 lists the values of the invariants of the two solitary waves with amplitudes $A_{1}=-2$, $A_{2}=1$. It can be seen that the values obtained for the invariants are satisfactorily constant during the computer run.

\section{The Maxwellian Initial Condition}

As a last study, we consider here is the numerical solution of the Equation (1) with the Maxwellian initial condition

$$
U(x, 0)=\mathrm{e}^{-x^{2}}
$$

with the boundary conditions

$$
U(-20, t)=U_{x}(-20, t)=U(20, t)=U_{x}(20, t)=0 .
$$

As it is known, Maxwellian initial condition (14) the behavior of the solution, depends on the values of $\mu$. So we have considered various values for $\mu$. For the first linearization technique the computations are carried out for the cases $\mu=1,0.5,0.1,0.05,0.02$ and 0.005 which are used in the earlier papers [5,14]. When $\mu=1,0.5$ is used as shown Figures 4(a) and (b) at time $t=12$ the Maxwellian initial condition does not cause development into a clean solitary wave. However with smaller values of $\mu=0.1,0.05,0.02$ and 0.005 Max-

\begin{tabular}{|c|c|c|c|c|}
\hline \multicolumn{5}{|c|}{$A_{1}=-2, \quad A_{2}=1$} \\
\hline$t$ & Lineerization & $C_{1}$ & $C_{2}$ & $C_{3}$ \\
\hline 0 & & -3.1415739 & 13.3332981 & 22.6665313 \\
\hline 5 & & -3.1373324 & 13.3219118 & 22.6210653 \\
\hline 15 & & -3.1227097 & 13.2806152 & 22.4483653 \\
\hline 25 & First & -3.1143337 & 13.2581754 & 22.3589494 \\
\hline 35 & & -3.1060334 & 13.2359744 & 22.2706624 \\
\hline 45 & & -3.0978106 & 13.2140028 & 22.1834701 \\
\hline 55 & & -3.0896638 & 13.1922565 & 22.0973499 \\
\hline 0 & & -3.1415739 & 13.3332981 & 22.6665313 \\
\hline 5 & & -3.1391704 & 13.3196543 & 22.6120524 \\
\hline 15 & & -3.1325076 & 13.2805526 & 22.4637733 \\
\hline 25 & Second & -3.1277388 & 13.2535359 & 22.3561477 \\
\hline 35 & & -3.1230138 & 13.2268810 & 22.2501653 \\
\hline 45 & & -3.1183416 & 13.2005566 & 22.1457584 \\
\hline 55 & & -3.1137210 & 13.1745553 & 22.0428880 \\
\hline
\end{tabular}

Table 3. Invariants for interaction of two solitary wave. wellian initial condition breaks up into more solitary waves which were drawn in Figures 4(c)-(f) at time $t=12$. The numerical conserved quantities with $\mu=1,0.5,0.1,0.05,0.02$ and 0.005 are given in Table 4 . It is observed that the obtained values of the invariants remain almost constant during the computer run.

Table 4. Invariants for Maxwellian initial condition, differ-

\begin{tabular}{|c|c|c|c|c|}
\hline$t$ & $\mu$ & $C_{1}$ & $C_{2}$ & $C_{3}$ \\
\hline 0 & & 1.772454 & 2.506607 & 0.886227 \\
\hline 3 & & 1.772972 & 2.506836 & 0.886561 \\
\hline 6 & 1 & 1.775116 & 2.512628 & 0.890240 \\
\hline 9 & & 1.776365 & 2.514942 & 0.891795 \\
\hline 12 & & 1.776698 & 2.515136 & 0.891967 \\
\hline 0 & & 1.772454 & 1.879971 & 0.886227 \\
\hline 3 & & 1.772452 & 1.879970 & 0.886225 \\
\hline 6 & 0.5 & 1.772451 & 1.879968 & 0.886224 \\
\hline 9 & & 1.772451 & 1.879967 & 0.886223 \\
\hline 12 & & 1.772450 & 1.879966 & 0.886222 \\
\hline 0 & & 1.772454 & 1.378646 & 0.886226 \\
\hline 3 & & 1.772420 & 1.378591 & 0.886174 \\
\hline 6 & 0.1 & 1.772368 & 1.378507 & 0.886054 \\
\hline 9 & & 1.772316 & 1.378424 & 0.885933 \\
\hline 12 & & 1.772264 & 1.378340 & 0.885813 \\
\hline 0 & & 1.772454 & 1.315980 & 0.886227 \\
\hline 3 & & 1.772266 & 1.315654 & 0.885789 \\
\hline 6 & 0.05 & 1.771976 & 1.315150 & 0.884947 \\
\hline 9 & & 1.771685 & 1.314648 & 0.884107 \\
\hline 12 & & 1.771396 & 1.314147 & 0.883270 \\
\hline 0 & & 1.772454 & 1.278380 & 0.886227 \\
\hline 3 & & 1.770834 & 1.275265 & 0.880933 \\
\hline 6 & 0.02 & 1.768546 & 1.271572 & 0.874402 \\
\hline 9 & & 1.766186 & 1.266707 & 0.864400 \\
\hline 12 & & 1.763931 & 1.262351 & 0.855782 \\
\hline 0 & & 1.772454 & 1.259581 & 0.886227 \\
\hline 3 & & 1.757684 & 1.254254 & 0.928815 \\
\hline 6 & 0.005 & 1.738212 & 1.227138 & 0.880527 \\
\hline 9 & & 1.722397 & 1.171836 & 0.714373 \\
\hline 12 & & 1.710490 & 1.162646 & 0.737751 \\
\hline
\end{tabular}
ent $\mu$. 


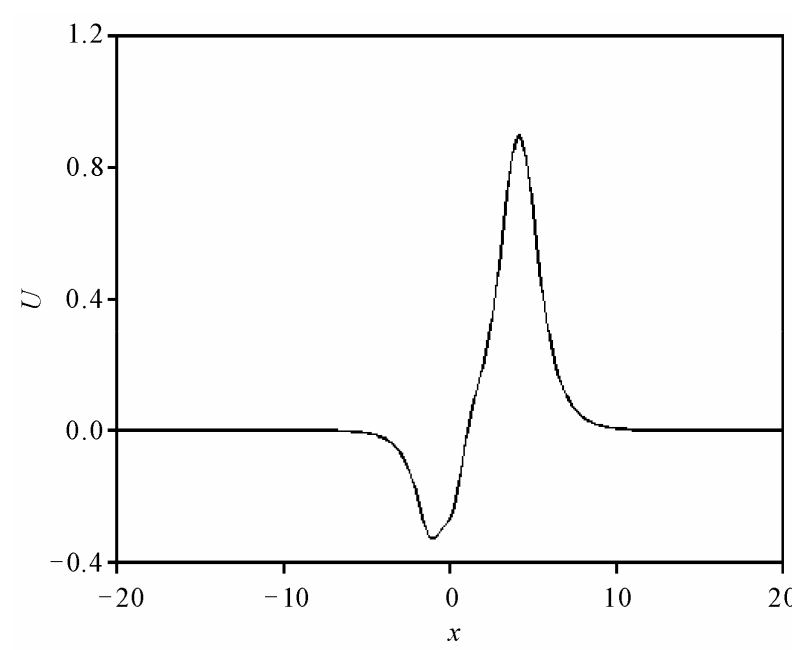

(a)

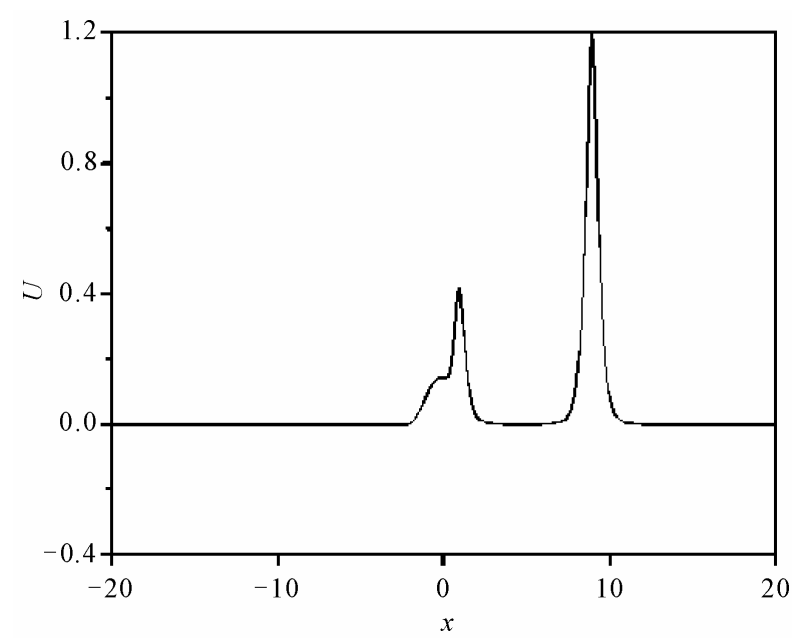

(c)

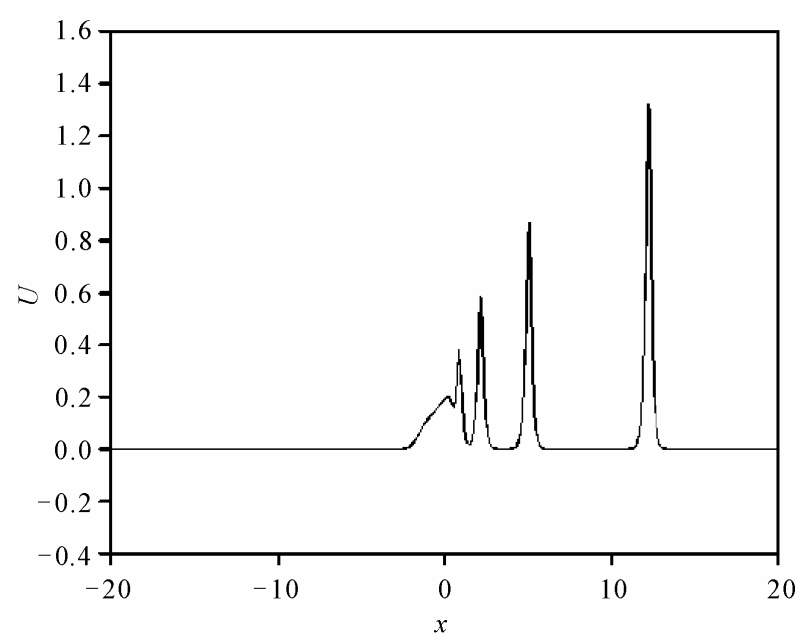

(e)

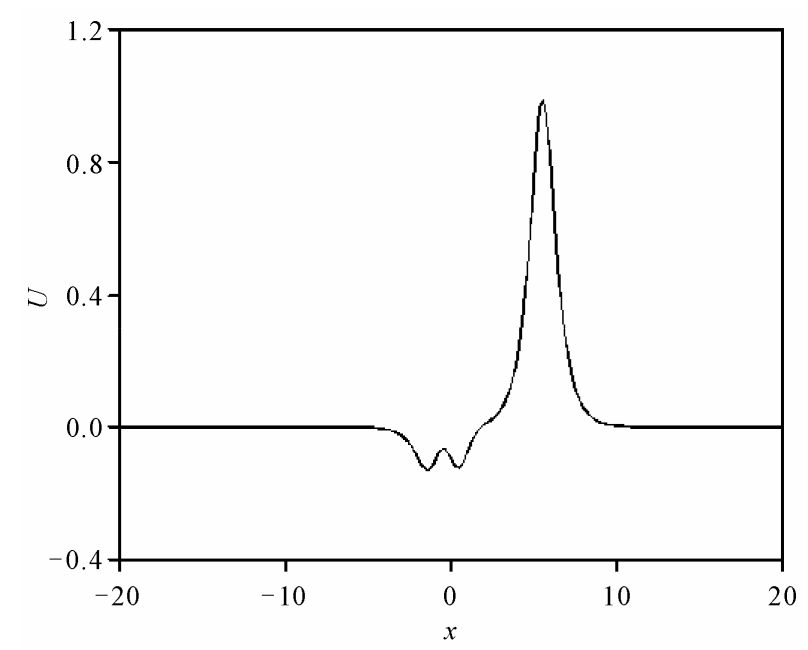

(b)

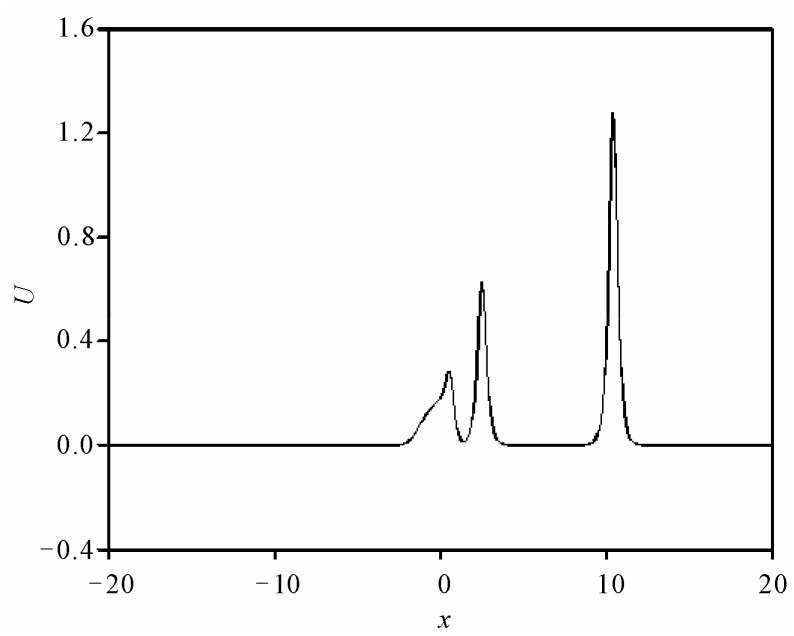

(d)

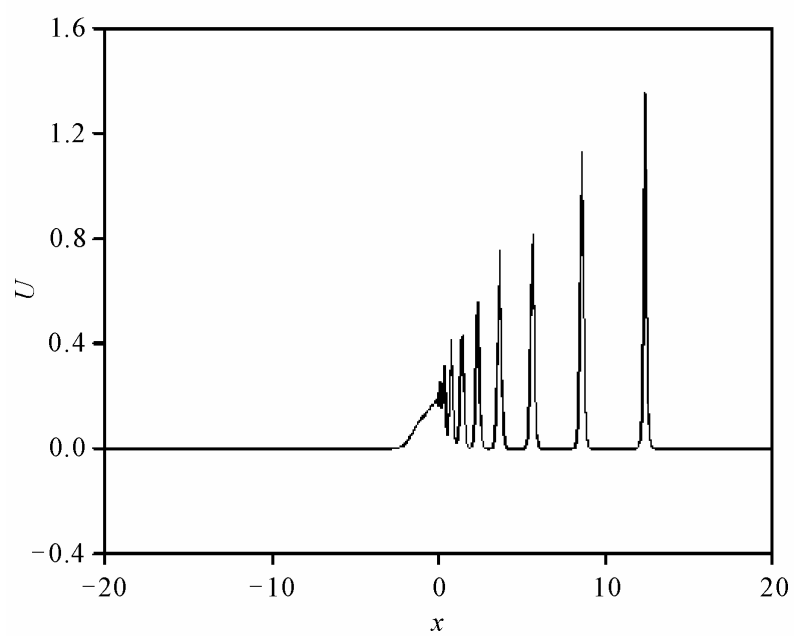

(f)

Figure 4. Maxwellian initial condition, state at time $t=12$ (a) $\mu=1$, (b) $\mu=0.5$, (c) $\mu=0.1$, (d) $\mu=0.05$, (e) $\mu=0.02$, (f) $\mu=$ 0.005 . 


\section{Conclusions}

In this study, a numerical solution of the MEW equation based on the septic B-spline finite element has been presented with three different linearization techniques. Three test problems are worked out to examine the performance of the algorithms. The performance and accuracy of the method were demonstrated by calculating the error norms $L_{2}$ and $L_{\infty}$ on the motion of a single solitary wave. For the first and second linearization techniques, the error norms are sufficiently small and the invariants are satisfactorily constant in all computer run. The obtained results from the first and the second linearization techniques are almost the same and the computed results show that the present method is a remarkably successful numerical technique for solving the MEW equation and can also be efficiently applied to other types of non-linear problem.

\section{References}

[1] P. J. Morrison, J. D. Meiss and J. R. Carey, "Scattering of Regularized-Long-Wave Solitary Waves," Physica D: Nonlinear Phenomena, Vol. 11, No. 3, 1984, pp. 324-336. doi:10.1016/0167-2789(84)90014-9

[2] L. R. T. Gardner and G. A. Gardner, "Solitary Waves of the Regularized Long Wave Equation," Journal of Computational Physics, Vol. 91, No. 2, 1990, pp. 441-459. doi:10.1016/0021-9991(90)90047-5

[3] L. R. T. Gardner and G. A. Gardner, "Solitary Waves of the Equal Width Wave Equation," Journal of Computational Physics, Vol. 101, No. 1, 1992, pp. 218-223. doi:10.1016/0021-9991(92)90054-3

[4] L. R. T. Gardner, G. A. Gardner, F. A. Ayoup and N. K. Amein, "Simulations of the EW Undular Bore," Communications in Numerical Methods in Engineering, Vol. 13, No. 7, 1997, pp. 583-592. doi:10.1002/(SICI)1099-0887(199707)13:7<583::AID-C NM90>3.0.CO;2-E

[5] S. I. Zaki, "Solitary Wave Interactions for the Modified Eual Width Equation," Computer Physics Communications, Vol. 126, No. 3, 2000, pp. 219-231. doi:10.1016/S0010-4655(99)00471-3

[6] S. I. Zaki, "A Least-Squares Finite Element Scheme for the Ew Equation," Computer Methods in Applied Mechanics and Engineering, Vol. 189, No. 2, 2000, pp. 587594. doi:10.1016/S0045-7825(99)00312-6

[7] A. M. Wazwaz, "The tanh and sine-cosine Methods for a Reliable Treatment of the Modified Equal Width Equation and Its Variants," Communications in Nonlinear Science and Numerical Simulation, Vol. 11, No. 2, 2006, pp. 148-160. doi:10.1016/j.cnsns.2004.07.001
[8] A. Esen, "A Numerical Solution of the Equal Width Wave Equation by a Lumped Galerkin Method," Applied Mathematics and Computational, Vol. 168, No. 1, 2005, pp. 270-282. doi:10.1016/j.amc.2004.08.013

[9] A. Esen, "A Lumped Galerkin Method for the Numerical Solution of the Modified Equal Width Wave Equation Using Quadratic B Splines," International Journal of Computer Mathematics, Vol. 83, No. 5-6, 2006, pp. 449459. doi:10.1080/00207160600909918

[10] B. Saka, "Algorithms for Numerical Solution of the Modified Equal Width Wave Equation Using Collocation Method," Mathematical and Computer Modelling, Vol. 45, No. 9-10, 2007, pp. 1096-1117. doi:10.1016/j.mcm.2006.09.012

[11] B. Saka and İ. Dag, "Quartic B-Spline Collocation Method to the Numerical Solutions of the Burgers Equation," Chaos, Solitons and Fractals, Vol. 32, No. 3, May 2007, pp. 1125-1137. doi:10.1016/j.chaos.2005.11.037

[12] J. F. Lu, "He's Variational Method for the Modified Equal Width Wave Equation," Chaos, Solitons and Fractals, Vol. 39, No. 5, 2009, pp. 2102-2109. doi:10.1016/j.chaos.2007.06.104

[13] S. Hamdi, W. E. Enright, W. E. Schiesser, J. J. Gottlieb and A. Alaal, "Exact Solutions of the Generalized Equal Width Wave Equation," Proceedings of the International Conference on Computational Science and Its Application, LNCS, Springer-Verlog, Berlin, Vol. 2668, 2003, pp. 725-734.

[14] D. J. Evans, K. R. Raslan and A. Alaal, "Solitary Waves for the Generalized Equal Width (Gew) Equation," International Journal of Computer Mathematics, Vol. 82, No. 4, 2005, pp. 445-455.

[15] T. Geyikli, "Modelling Solitary Waves of a Fifth-Order Non-Linear Wave Equation," International Journal of Computer Mathematics, Vol. 84, No. 7, July 2007, pp. 1079-1087. doi:10.1080/00207160701294368

[16] S. G. Rubin and R. A. Graves, "A Cubic Spline Approximation for Problems in Fluid Mechanics," NASA TR R-436, Washington, DC, 1975.

[17] J. Caldwell and P. Smith, "Solution of Burgers' Equation with a Large Reynolds Number," Applied Mathematical Modelling, Vol. 6, No. 5, October 1982, pp. 381-386. doi:10.1016/S0307-904X(82)80102-9

[18] A. Esen and S. Kutluay, "Solitary Wave Solutions of the Modified Equal Width Wave Equation," Communications in Nonlinear Science and Numerical Simulation, Vol. 13, No. 8, 2008, pp. 1538-1546. doi:10.1016/j.cnsns.2006.09.018

[19] K. R. Raslan, "Collocation Method Using Cubic B-Spline for the Generalized Equal Width Equation," International Journal of Simulation and Process Modelling, Vol. 2, No. 1-2, 2006, pp. 37-44. 